\title{
SEMISIMPLE BANDS
}

\author{
BY \\ B. D. ARENDT
}

A band is a semigroup in which every element is idempotent. A right congruence $\tau$ on a semigroup $S$ is said to be modular if there exists an element $e$ in $S$ such that $(e x) \tau x$ for all $x$ in $S$. The symmetric definition holds for left congruences, and a two-sided congruence is said to be modular if it is modular both as a left and a right congruence and hence has a two-sided identity.

Following Oehmke [7] (see also [3]) we define the radical $R_{r}\left(R_{l}, R_{t}\right)$ of a band $S$ to be the intersection of all the maximal, modular right (left, two-sided) congruences on $S . S$ is said to be $x$-semisimple ( $x$-radical) if the radical $R_{x}$ is the identity relation ¿ (universal relation $v$ ) on $S$.

There are three distinct types of maximal, modular right congruences on a band. This classification enables us to prove that a band is $r$-semisimple if and only if it is a semilattice $\Gamma$ of right zero semigroups $S_{y}$ such that for $x, y \in S_{y}, x \neq y$, the principal left ideals $S x$ and $S y$ are disjoint.

A band has only one type of maximal, modular two-sided congruence, and this is used to show that a band $S$ is $t$-semisimple if and only if it is a semilattice.

Kimura [5, Lemma 1] has characterized a rectangular band as the cartesian product of two sets with multiplication given by $(a, b)(c, d)=(a, d)$. The structure theory for arbitrary bands begins with the following result by McLean [6, Theorem $1]$.

THEOREM 1. Let $S$ be a band. Then there exists a unique two-sided congruence $\mu$ on $S$ whose equivalence classes are rectangular bands and such that $S / \mu$ is a semilattice. If $\sigma$ is any two-sided congruence on $S$ such that $S / \sigma$ is a semilattice, then $\mu \leqq \sigma$.

It remains to determine what Clifford and Preston [1, p. 25] have called the "fine structure" of an arbitrary band, that is, how the elements of the different rectangular bands multiply together. Using the characterization given above we are able to describe this internal structure of those bands which are $r$-semisimple. A comparison is also made with some recent results by Howie [4]. Finally we characterize those bands which are a semilattice of right (left) zero semigroups.

Let $\tau$ be a right congruence on a semigroup $S$. For any $a$ in $S$ we define an equivalence relation $\tau a$ on $S$ by $x(\tau a) y$ if and only if $(a x) \tau(a y)$. Then $\tau a$ is a right congruence on $S$ and Oehmke has shown [7, Lemma 4] that $a \tau b$ implies $\tau a=\tau b$.

Received by the editors October 21, 1968. 
When there is no danger of ambiguity we will denote the equivalence class of a fixed congruence $\tau$ that contains an element $a$ of $S$ by $S_{a}$.

For completeness we include the following results of Oehmke [7].

Lemma 2 [7, Lemma 6]. Let $S$ be a semigroup, $\tau$ a right congruence on $S$ and $S^{*}$ the union of a set of equivalence classes of $\tau$. Define arb if and only if for every $c$ in $S$ we have ac $\in S^{*}$ if and only if $b c \in S^{*}$. Then $\sigma$ is a right congruence on $S$ such that $\tau \leqq \sigma$.

LEMMA 3 [7, LEMMA 7]. If $\sigma$ is a maximal right congruence and $R$ any right ideal of $S$, then either $R$ is contained in an equivalence class of $\sigma$ (which is also a right ideal of $S$ ) or $R$ contains an element of each equivalence class.

LEMMA 4 [7, THEOREM 9]. If $\tau$ is a maximal, modular right congruence on the semigroup $S$, then either ta is a maximal, modular right congruence on $S$ or $S_{a}$ is a right ideal and $\tau a=\nu$.

Since the results for left and right congruences are similar due to the symmetry of the definitions, we consider only the latter. For definitions and terminology not otherwise explained the reader is referred to [1] and [7].

This paper contains the results of the author's dissertation written at the University of Iowa. The author wishes to express his gratitude to Professor R. H. Oehmke who directed this dissertation.

1. Congruences on bands. The proofs of the first six results are straightforward and are omitted. The first is perhaps the most useful arising from the hypothesis that $x^{2}=x$ for all $x$ in $S$.

LEMMA 5. If $\tau$ is a right congruence on a band $S$, then the equivalence classes of $\tau$ are subbands of $S$.

It is easy to see that $a$ is a left identity for the right congruence $\tau a$ in a band $S$ and therefore $\tau a$ is modular for all $a$ in $S$. A natural question at this point is the nature of $\tau e$ where $e$ is a left identity for $\tau$, for which we have the following answer.

LEMMA 6. Let a be an element of the band $S$ with $\tau$ a right congruence on $S$. Then $\tau a=\tau$ if and only if $a$ is a left identity for $\tau$.

Thus for a right congruence $\tau$ on a band $S$, the modularity of $\tau$ is related to the nature of the right congruences $\tau a$. We note that the "if" part of Lemma 6 holds for an arbitrary semigroup.

Another question of interest is the relation of a right congruence $\tau$ to the ideal structure of $S$, and again we find the answer related to the nature of the congruences ra.

THEOREM 7. If $\tau$ is a right congruence on a band $S$, then an equivalence class $S_{a}$ of $\tau$ is a right ideal of $S$ if and only if for all $b$ in $S_{a}, \tau b=\nu$. 
For a fixed congruence $\tau$ we introduce the notation $I=I_{\tau}=\{a \in S: \tau a=\nu\}$. Then Theorem 7 states that an equivalence class $S_{a}$ of $\tau$ is a right ideal if and only if $S_{a} \subset I$. Similarly, we denote $E=E_{\tau}=\{a \in S: \tau a=\tau\}$, the set of left identities for $\tau$. Either $E$ or $I$ may be empty.

THEOREM 8. If $\tau$ is a right congruence on a band $S$ and an equivalence class $S_{a}$ of $\tau$ is a two-sided ideal of $S$, then $S_{a}=I$.

LEMMA 9. Let $\tau$ be a modular right congruence on a band $S$. If $x y$ is a left identity for $\tau$ then so is $y$.

We are now prepared to describe the maximal, modular right congruences on a band.

THEOREM 10. Let $S$ be a band and $\tau$ a modular right congruence on $S$. Let $E=\{a: \tau a=\tau\}$. The congruence $\tau$ is a maximal modular right congruence if and only if $\tau$ has exactly two equivalence classes and one of the following three alternatives holds:

1. $S=E$. In this case the congruence classes are both left ideals of $S$.

2. $S \neq E$ and $I=\{a: \tau a=\nu\} \neq \varnothing$. Then $E$ and $I$ are the congruence classes for $\tau$.

3. $S \neq E$ and $I=\varnothing$. In this case the congruence classes are $E$ and the left ideal $T=\{a \notin E: a e \in E$ for some $e \in E\}$.

Proof. It is straightforward to show that either $E=S$ or $E$ is a congruence class for $\tau$. Suppose $E=S$. Then each congruence class is a left ideal, and if $\tau$ had more than two congruence classes the equivalence relation formed by taking the union of two of them would be a right congruence strictly larger than $\tau$.

Suppose now that $E \neq S$ so that $E$ is a congruence class for $\tau$. Define $T$ as above and let $U=S-(E \cup T)$. If we assume $I \neq \varnothing$ then $U \neq \varnothing$, and using Lemma 9 and Theorem 8 we conclude $U=I$ is a congruence class for $\tau$ which is a two-sided ideal. For each $a \in S$ define $R_{a}=\{x: a x \in I\}$. Each of these sets is a right ideal of $S$ containing $I$. Define an equivalence relation $\sigma$ on $S$ by $a \sigma b$ if and only if $R_{a}=R_{b}$. By Lemma $2 \sigma$ is a right congruence with $\tau \leqq \sigma$ so $\sigma=\tau$ or $\sigma=\nu$. If $\sigma=\nu$ then since there exists an $a \in I$ we have $S=R_{a}=R_{e}=I$ for $e$ in $E$, a contradiction. Thus $\sigma=\tau$ and $R_{a}=R_{b}$ if and only if $a \tau b$. If $S$ contains an element $x$ not in $E \cup I$ then $S_{x} \cap R_{x}=\varnothing$ and the right ideal $R_{x}$ is contained in a congruence class for $\tau$ by Lemma 3. But $I \subset R_{x}$ so $R_{x}=I=R_{e}$ for $e$ in $E$, and $x \tau e$, a contradiction. Thus $S=E \cup I$.

It remains to consider the case where $E \neq S$ and $I=\varnothing$, so $S=E \cup T$. We first prove the following lemma.

LEMMA 11. Let $\tau$ be a maximal, modular right congruence on a band $S$, then $(x y x) \tau(y x)$ for all $x, y$ in $S$.

Proof. The conclusion is immediate for the first two types of congruences, so assume $S=E \cup T$. For each $a$ in $S$ define $E_{a}=\{x: a x \in E\}$ and $a \sigma b$ if and only if 
$E_{a}=E_{b}$. By Lemma $2 \sigma$ is a right congruence with $\tau \leqq \sigma . \sigma=\nu$ implies $\tau$ is of type 1 so we conclude $\sigma=\tau$ and $E_{a}=E_{b}$ if and only if $a \tau b$. From Lemma $9 E_{y x} \subset E_{x}$ so $E_{y x}=E_{y x y x} \subset E_{x y x} \subset E_{y x}$ implying $(x y x) \tau(y x)$.

Returning to the proof of the theorem we want to show first that each equivalence class in $T$ is a left ideal. Let $a \in T$ and consider the congruence $\tau a$. By Lemmas 4 and 6 we know that $\tau a$ is a maximal, modular right congruence different from $\tau$ so that the congruence $\tau \cup \tau a=\nu$. Thus [2, Lemma 1] for any $y$ in $S$ there is a finite sequence $x_{1}, \ldots, x_{n}$ such that $a(\tau a) x_{1} \tau x_{2}(\tau a) x_{3} \tau x_{4} \cdots \tau y$ where we may select the particular order of the congruences $\tau$ and $\tau a$ without loss of generality. Now $a(\tau a) x_{1}$ implies $a \tau\left(a x_{1}\right)$ which gives $a \tau\left(a x_{1} a\right)$. By Lemma 11 we get $a \tau\left(x_{1} a\right)$, so that $a \tau\left(a x_{2} a\right)$ or $a(\tau a) x_{2} a$. But then $a(\tau a) x_{3} a$ and continuing in this manner we obtain $a(\tau a) y a$ so $a \tau(a y a)$. Using Lemma 11 again gives $a \tau(y a)$ for all $y$ in $S$ so $S_{a}$ is a left ideal.

Finally let $a$ and $b$ be elements of $T . E_{a}$ is easily shown to be a congruence class for the maximal, modular right congruence $\tau a$, and since it is not the class of left identities, $E_{a}$ is a left ideal. Thus for $e$ in $E_{a}$ we have $b e(\tau a) e$ which implies $(a b e) \tau(a e) \tau e$ so $E_{a} \subset E_{a b}$. But $E_{a b} \subset E_{b}$ by Lemma 9 implying $E_{a} \subset E_{b}$. By symmetry $E_{b} \subset E_{a}$ so $a \tau b$ and $T$ is a congruence class for $\tau$. The converse is obvious, concluding the proof of the theorem.

TheOREM 12. Let $S$ be a band. A congruence $\tau$ on $S$ is a maximal, modular twosided congruence if and only if $\tau$ has exactly two equivalence classes, $E=\{a: \tau a=\tau\}$ and $I=\{a: \tau a=\nu\}$.

Proof. Let $\tau$ be a maximal, modular two-sided congruence. If $\tau \leqq \sigma$ where $\sigma$ is a maximal, modular right congruence, then $\sigma$ is not of type 1 since it could not have a two-sided identity. Suppose $\sigma$ is of type 3 and let $a \in T$. Then $\tau=\sigma \cap \sigma a$ by [7, Lemmas 2, 3 and 5] so $x \sigma y$ if and only if $x \tau y$ and $x(\tau a) y$, implying that $F=E-E_{a}$, $E_{a}$ and $T$ are the congruence classes for $\tau$. Moreover $F$ must be the class of identities for $\tau$ so that $F E_{a} \subset E_{a}$. Let $V=E \cup E_{a}$ and $\mu$ be the equivalence relation given by $S=F \cup V$. Then $\mu$ is a modular two-sided congruence strictly greater than $\tau$, a contradiction. Thus $\sigma$ is of type 2 and $\tau=\sigma$.

On the other hand, if $\tau$ is not contained in a maximal, modular right congruence then as we shall see in Theorem $15, S / \tau$ is a left zero semigroup. But $S / \tau$ has an identity so it must have only one element, contradicting the maximality of $\tau$.

2. The radical of a band. We now want to characterize those bands for which $R_{r}$ or $R_{t}$ is the universal relation $\nu$. Oehmke has shown that in general these radicals are two-sided congruences [7, Lemma 19] and that the semigroup modulo the radical has a trivial radical $\iota$ [7, Theorem 21]. Moreover it follows from the proof of Theorem 12 that $R_{r} \leqq R_{t}$.

LEMMA 13. Let $S$ be a band, then $a b R_{t} b a$ for all $a, b$ in $S$. 
Proof. Let $\tau$ be any maximal modular two-sided congruence on $S$, and $a, b \in S$. Since $\tau$ induces a decomposition $S=E \cup I$, it follows directly by case considerations that $a b \tau b a$ and therefore $a b R_{t} b a$.

THEOREM 14. Let $S$ be a band, then $R_{t}=\nu$ if and only if $S$ is rectangular.

Proof. Suppose $S$ is rectangular, $S=A \times B$, and $(a, b),(c, d) \in S$. Then $(a, b)=$ $(a, y)(x, b)$ and by Lemma $13(a, y)(x, b) R_{t}(x, b)(a, y)=(x, y)$. Similarly $(x, y) R_{t}(c, d)$ so $(a, b) R_{t}(c, d)$ and $R_{t}=\nu$. Conversely suppose that $R_{t}=\nu$. If the congruence $\mu$ of Theorem 1 equals $\nu$ then we are finished; so suppose not, and let $S_{a}$ and $S_{b}$ be distinct congruence classes of $\mu$. By the way the multiplication of $S$ behaves (Theorem 1) it is easy to see that any congruence on the underlying semilattice generates a congruence on $S$. But as we shall see in the proof of Theorem 16 this means there exists a maximal modular two-sided congruence separating $S_{a}$ and $S_{b}$ so we cannot have $a R_{t} b$, a contradiction. Therefore $\mu=\nu$ and we are done.

Finally we characterize those bands for which the right radical is equal to $\nu$.

THEOREM 15. Let $S$ be a band, then $R_{r}=v$ if and only if $S$ is a left zero semigroup.

Proof. If $S$ is a left zero semigroup it has no proper left ideals. On the other hand, any maximal modular right congruence has at least one equivalence class which is a left ideal so we must have $R_{r}=\nu$. Conversely if $R_{r}=\nu$ then $R_{t}=\nu$ and by Theorem $14 S$ is a rectangular band $A \times B$. If $B$ has more than one element then the disjoint subsets $A \times\{b\}$ and $A \times(B-\{b\})$ form a maximal modular right congruence contradicting $R_{r}=\nu$. Thus $B=\{b\}$ and $S$ is a left zero semigroup.

3. Semisimple bands. We are now ready to characterize the semisimple bands.

THEOREM 16. Let $S$ be a band, then $S$ is t-semisimple if and only if $S$ is a semilattice.

Proof. Suppose $S$ is $t$-semisimple. By Lemma 13 for all $a, b$ in $S$ we have $a b R_{t} b a$ so that $R_{t}=\iota$ implies $a b=b a$ and $S$ is a semilattice.

Conversely suppose $S$ is a semilattice. If $S$ has only one element it is $t$-semisimple; so suppose there exist $a, b$ in $S$ with $a \neq b$. Define an equivalence relation $\tau_{a}$ on $S$ as follows. Let $E(a)=\{x: x a=a\}$ and $I(a)=S-E(a)$. Then $E(a)$ is a subband and $I(a)$ is an ideal so by Theorem $12 \tau_{a}$ is a maximal, modular two-sided congruence if both $E(a)$ and $I(a)$ are not empty. If $b \in I(a)$ that condition is satisfied and the congruence $\tau_{a}$ separates $a$ and $b$. On the other hand, if $b \in E(a)$ then the corresponding congruence $\tau_{b}$ separates $a$ and $b$ so $S$ must be $t$-semisimple.

COROLlary 17. Let $S$ be a band, then the congruence $R_{t}$ on $S$ is equal to the congruence $\mu$ of Theorem 1 .

THeOREM 18. Let $S$ be a band. By Theorem 1, $S=\bigcup\left\{S_{\gamma}: \gamma \in \Gamma\right.$, a semilattice $\}$, where each $S_{\gamma}$ is a rectangular band. Then $S$ is r-semisimple if and only if each $S_{\gamma}$ is a right zero semigroup such that if $x, y \in S_{y}, x \neq y$, then $S x \cap S y=\varnothing$. 
Proof. Suppose $S$ is $r$-semisimple and write each rectangular band $S_{\gamma}=A_{\gamma} \times B_{\gamma}$. Suppose further that for some $\gamma \in \Gamma$ there exist $a, a^{\prime}$ in $A_{\gamma}$ with $a \neq a^{\prime}$. Since $R_{r}=\iota$ there exists maximal modular right congruence $\tau$ such that $(a, b) \not \equiv\left(a^{\prime}, b\right)(\bmod \tau)$. By Lemma $11(a, b)=(a, b)\left(a^{\prime}, b\right)(a, b) \tau\left(a^{\prime}, b\right)(a, b)=\left(a^{\prime}, b\right)$, a contradiction. We conclude that $A_{\gamma}$ contains only one element for all $\gamma \in \Gamma$, and $S_{\gamma}$ is a right zero semigroup.

Now suppose that $x, y$ are in some $S_{y}$ with $x \neq y$ so there exists a maximal modular right congruence $\tau$ on $S$ such that $x \not \equiv y(\bmod \tau)$. Then $\tau$ cannot be of type 2 , and if $\tau$ is of type 1 the conclusion follows immediately. Assume $\tau$ has the form $S=E \cup T$ with $x$ in $E$ and $y$ in $T$. If $S x \cap S_{y} \neq \varnothing$, then there exists an element $z$ such that $(z x) \tau y$ holds. Thus $(y z x) \tau y$ by Lemma 5 so that $z x(\tau y) y$. Applying Lemma 9 to the congruence $\tau y$ we have $x(\tau y) y$ which gives $(y x) \tau y$. But $y x=x$ giving a contradiction, so $S x \cap S_{y}=\varnothing$ implying $S x \cap S y=\varnothing$ in this case also.

Conversely, if $x$ and $y$ are distinct elements of $S$ we want to exhibit a maximal, modular right congruence separating them. From the proof of Theorem 16 we may assume that $x$ and $y$ are in the same $S_{\gamma}$. Let $L_{1}$ be the union over all subsets of $S$ of the form $S z$ such that $S z \cap S x \neq \varnothing$. Both $L_{1}$ and its complement $L_{2}$ are nonempty left ideals which gives us the desired congruence and $S$ must be $r$-semisimple.

COROllaRy 19. A right zero semigroup is r-semisimple.

The above corollary together with the fact that a left zero semigroup has $R_{r}=\nu$ might lead one to suspect that the condition $S x \cap S y=\varnothing$ for $x \neq y$ in the same $S_{\gamma}$ is redundant. The following example shows that this is not the case. Let $\Gamma=\{0,1\}$ with the usual multiplication and let $S_{1}=\{a, b\}$ and $S_{0}=\{c, d\}$ be right zero semigroups. Define $a c=c a=b c=c b=c, a d=b d=d$, and $d a=d b=c$. One may verify that the multiplication is associative so that $S=S_{0} \cup S_{1}$ is a band. Moreover $S a=\{a, c\}$ while $S b=\{b, c\}$ so that $S a \cap S b \neq \varnothing$. Thus the $r$-semisimplicity of $S$ depends not only on the fact that $S$ is a semilattice $\Gamma$ of right zero semigroups $S_{y}$, but also on how the elements of the different $S_{y}$ multiply together. It is this problem that we consider next.

4. $r$-semisimple bands with simply ordered semilattice. If the band

$$
S=\bigcup\left\{S_{\gamma}: \gamma \in \Gamma, \text { a semilattice }\right\}
$$

then the partial ordering on $\Gamma$ gives a partial ordering on the collection of right zero semigroups $S_{\gamma}$. Thus we will write $S_{\gamma} \geqq S_{\delta}$ when $\gamma \geqq \delta$ for $\gamma, \delta \in \Gamma$. If $S_{\gamma}$ and $S_{\delta}$ are ordered this way and $x \in S_{y}, y \in S_{\delta}$ then it is clear from Theorem 1 that $x y$ and $y x$ both lie in $S_{\delta}$. In fact Dean and Oehmke [2, p. 1195] have shown that we always have $x y=y$.

Our objective now is to determine the possibilities for constructing $r$-semisimple bands out of right zero semigroups. In the discussion and results that follow it will be convenient to drop the subscript notation $S_{\gamma}$ and use the letters $A, B, C, \ldots$ to 
denote the right zero semigroups of the band $S$. We will first determine the construction of semisimple bands whose semilattice $\Gamma$ is simply ordered, and then the general case.

LEMMA 20. Let the band $S$ be a semilattice of right zero semigroups as in Theorem 1. If $A$ and $B$ are two of these right zero semigroups with $A>B$, then $A$ induces $a$ two-sided congruence $\tau_{A B}$ on $B$.

Proof. We remark that any equivalence relation on a right zero semigroup is a two-sided congruence and any mapping of one right zero semigroup into another is a homomorphism. Thus the mapping $b \rightarrow b a$ induces a two-sided congruence $\tau_{a}$ on $B$ for every $a$ in $A$. Furthermore it is straightforward to show that $\tau_{a}=\tau_{a^{\prime}}$ for all $a, a^{\prime}$ in $A$ and we denote this congruence by $\tau_{A B}$.

THEOREM 21. Let $S$ be a simply ordered semilattice of right zero semigroups. Then $S$ is an r-semisimple band if and only if the following hold.

(1) For each pair of right zero semigroups $A>B$ in $S$ we have a two-sided congruence $\tau_{A B}$ on $B$ with congruence classes $U_{i}(A, B), i$ in some index set.

(2) For each $i$ we have a monomorphism $\phi_{i}(A, B): A \rightarrow U_{i}(A, B)$ with multiplication given by $a b=b$ and $b a=a \phi_{i}(A, B)$ for $a \in A, b \in U_{i}(A, B)$.

(3) For $A>B>C, \tau_{A C} \leqq \tau_{B C}$ and for each pair $U_{j}(B, C), U_{i}(A, B)$ there exists a $U_{k}(A, C) \subset U_{j}(B, C)$ such that $U_{j}(B, C) U_{i}(A, B) \subset U_{k}(A, C)$, and for this triple of indices, $\phi_{i}(A, B) \phi_{j}(B, C)=\phi_{k}(A, C)$.

Proof. Suppose $S$ is $r$-semisimple. (1) and (2) follow in an obvious way from Lemma 20. The fact that $\phi_{i}(A, B)$ is one-to-one is a consequence of the $r$-semisimplicity of $S$ by Theorem 18. $\tau_{A C} \leqq \tau_{B C}$ may be shown directly without the hypothesis that $S$ is $r$-semisimple and the rest of (3) comes from the associative law as follows. Let $c_{1} b_{1}, c_{2} b_{2} \in U_{j}(B, C) U_{i}(A, B)$. Then for $a$ in $A$ we have $\left(c_{1} b_{1}\right) a$ $=\left(c_{2} b_{1}\right) a=c_{2}\left(b_{1} a\right)=c_{2}\left(b_{2} a\right)=\left(c_{2} b_{2}\right) a$ so $\left(c_{1} b_{1}\right) \tau_{A C}\left(c_{2} b_{2}\right)$ and $c_{1} b_{1}, c_{2} b_{2} \in U_{k}(A, C)$ for some $k$. Also $a \phi_{i}(A, B) \phi_{j}(B, C)=(b a) \phi_{j}(B, C)$ for $b \in U_{i}(A, B)$, and $(b a) \phi_{j}(B, C)$ $=c(b a)$ for $c \in U_{j}(B, C)$. Since $c b \in U_{k}(A, C)$ we have $c(b a)=(c b) a=a \phi_{k}(A, C)$.

To prove the converse we need to show first that all possible products are associative and then, using the condition of Theorem 18, that $S$ is $r$-semisimple. Consider the product $x y z$ of three elements of $S$, assuming first that all three are in $A$ and $B$. If either $y$ or $z$ is in $B$ it is clearly associative, so consider $b a a^{\prime}$, where $b \in B$ and $a, a^{\prime} \in A . b \in U_{i}(A, B)$ for some $i$, thus $(b a) a^{\prime}=\left(a \phi_{i}(A, B)\right) a^{\prime}$ and since $a \phi_{i}(A, B) \in U_{i}(A, B),\left(a \phi_{i}(A, B)\right) a^{\prime}=a^{\prime} \phi_{i}(A, B)=b a^{\prime}=b\left(a a^{\prime}\right)$. Now suppose that $x, y$ and $z$ are in $A \cup B \cup C$. Associativity is immediate if either $y$ or $z$ is in $C$. Consider $c a b$. $c(a b)=c b=b \phi_{j}(B, C)$ where $c \in U_{j}(B, C)$. Also $c \in U_{k}(A, C) \subset U_{j}(B, C)$ for some $k$ so $(c a) b=\left(a \phi_{k}(A, C)\right) b=b \phi_{j}(B, C)$. Consider $c b a$. There exist $j, i$ such that $c \in U_{j}(B, C)$ and $b \in U_{i}(A, B)$ and associated with this pair is the unique set $U_{k}(A, C)$ of the hypotheses. Thus $c b=b \phi_{j}(B, C) \in U_{k}(A, C)$ and so $(c b) a=a \phi_{k}(A, C)$. On the other hand, $c(b a)=c\left(a \phi_{i}(A, B)\right)=a \phi_{i}(A, B) \phi_{j}(B, C)$ and associativity follows. 
That $S$ is semisimple is a straightforward consequence of the fact that the multiplication mappings are one-to-one.

From this theorem we see that the right zero semigroups of $S$ which are "above" a particular one, say $C$, in the semilattice, can be realized in terms of successively finer congruences on $C$. Thus starting with the congruence $C=\bigcup U_{j}(B, C)$, each $U_{j}$ contains an isomorphic copy of $B$, namely $B \phi_{j}(B, C) . B$ (and its copies in the $U_{j}$ ) contains the congruence $B=\bigcup U_{i}(A, B)$, and in turn each of these equivalence classes contains an isomorphic copy of $A$. The congruence $\tau_{A C}$ is obtained as an "extension" of the congruence induced isomorphically on each $B \phi_{j}(B, C) \subset U_{j}(B, C)$ by breaking up $U_{j}(B, C)-B \phi_{j}(B, C)$ into additional equivalence classes $U_{k}(A, C)$.

This process may be continued, either indefinitely or until the congruence $\iota$ is reached on $C$. Alternately, a congruence may be inserted between two given ones. The procedure can be extended in the other direction by isomorphically imbedding $C$ (and its congruences) in the equivalence classes of a congruence on a larger right zero semigroup $D$. It is easy to see that that part of the original semilattice in $S$ which lies above $C$ can be homomorphically mapped onto this semilattice of congruences on $C$. The mapping may not be an isomorphism because of the possibility that $\tau_{A C}=\tau_{B C}$, and when this happens we have the following result.

THEOREM 22. Let $S$ be an $r$-semisimple band with right zero semigroups $A>B>C$, then $\tau_{A C}=\tau_{B C}$ implies $\tau_{A B}=\nu$.

Proof. $\tau_{A C}=\tau_{B C}$ implies $U_{k}(A, C)$ is equal to the $U_{j}(B, C)$ containing it, and for any $i, U_{j}(B, C) U_{i}(A, B) \subset U_{k}(A, C)$. But by Theorem 18 , distinct $U_{i}(A, B)$ would have to go into distinct $U_{k}(A, C)$ under this multiplication so there can be only one equivalence class and $\tau_{A B}=\nu$.

In a recent paper Howie [4] has determined the structure of all bands which are naturally ordered. A band is said to be naturally ordered if $e \leqq f$ and $g \leqq h$ together imply $e g \leqq f h$, where $\leqq$ is the natural partial ordering on the band. The following theorem indicates how these naturally ordered bands fit into our present work.

THEOREM 23. Let $S$ be an $r$-semisimple naturally ordered band. If $A$ and $B$ are any two right zero semigroups of $S$ with $A>B$, then $\tau_{A B}=\nu$.

Proof. In his paper Howie has shown [4, p. 57] that for any $a$ in $A$ there exists exactly one $b$ in $B$ such that $b \leqq a$, that is $b a=a b=b$. This implies $B a=\{b\}$ since $S$ is $r$-semisimple. But $|B a|$ is exactly the number of equivalence classes of $\tau_{A B}$ so that $\tau_{A B}=\nu$.

5. The general case. We now want to consider the general case where the $r$-semisimple band $S$ is a semilattice $\Gamma$ of right zero semigroups $A, B, C, \ldots$ and where $\Gamma$ is not necessarily simply ordered. If $A$ and $B$ are not comparable under the induced partial ordering, then there exists a right zero semigroup $C$ such that $A B, B A \subset C$. It is the possibilities for the products $a b$ and $b a$ in $C$ that determine how the "branches" fit together and this is what we want to describe. We recall 
that for $A>C$ in the semilattice we have the congruence $\tau_{A C}$ of Lemma 20 with equivalence classes $U_{k}(A, C), k$ in some index set, and multiplication mappings $a \phi_{k}(A, C)=c a \in U_{k}(A, C)$ for any $a$ in $A$ and $c$ in $U_{k}(A, C)$. Since Lemma 20 holds for an arbitrary semilattice of right zero semigroups, it will be convenient to drop the hypothesis of $r$-semisimplicity at first.

THEOREM 24. Let $S$ be the union of a semilattice $\Gamma$ of right zero semigroups such that each simply ordered chain of right zero semigroups in $S$ forms a band. Then $S$ is $a$ band if and only if for each distinct triple $A, B, C$ of right zero semigroups with $A B, B A \subset C$ we have the following conditions satisfied:

(1) For each $a \in A$ there exists a $U_{j}(B, C)$ such that $C a \subset U_{j}(B, C)$.

(2) For each $b \in B$ there exists a $U_{k}(A, C)$ such that $C b \subset U_{k}(A, C)$. When (1) and (2) are satisfied we define $a b=b \phi_{j}(B, C)$ and $b a=a \phi_{k}(A, C)$.

Proof. If $S$ is a band and $a \in A, b \in B$, then $(C a) b=\{a b\}$ so $C a$ is contained in some $U_{j}(B, C)$. It is easy to see that $a b$ is that unique element of $U_{j}(B, C)$ which is fixed by right multiplication by $b$, that is $a b=b \phi_{j}(B, C)$. Similarly for $C b$ and $b a$.

For the converse we need only show associativity of products from $A, B$ and $C$ as given. Moreover the product $x y z$ is obviously associative if either $y$ or $z$ is in $C$. Consider products of the form $c a b$ and $c b a$. We have $(c a) b=b \phi_{j}(B, C)=a b=c(a b)$. Similarly, $(c b) a=c(b a)$. For products $x y z$ where none of these are in $C$ all are trivial except the types $a b a^{\prime}$ and $b a a^{\prime}$. Since $a b \in U_{k}(A, C),(a b) a^{\prime}=a^{\prime} \phi_{k}(A, C)=b a^{\prime}$ $=a\left(b a^{\prime}\right)$; and since $a \phi_{k}(A, C) \in U_{k}(A, C),(b a) a^{\prime}=\left(a \phi_{k}(A, C)\right) a^{\prime}=a^{\prime} \phi_{k}(A, C)=b a^{\prime}$ $=b\left(a a^{\prime}\right)$ so $S$ must be associative.

THEOREM 25. Let $S$ be a band. By Theorem $1 S$ is a semilattice of rectangular bands. Then $S$ is $r$-semisimple if and only if every simply ordered chain of rectangular bands in $S$ is r-semisimple.

Proof. We observe first that the hypothesis of $r$-semisimplicity implies that each of the rectangular bands is in fact a right semigroup. The only if part is obvious so let $A$ and $B$ be two right zero semigroups in $S$ which do not lie in some simply ordered chain. We continue the notation of Theorem 24 and define an equivalence relation on $A$ by $A_{j}=\left\{a \in A: C a \subset U_{j}(B, C)\right\}$. If $A_{j} \neq \varnothing$ then $A_{j} b \cap A_{j} b^{\prime}=\varnothing$ for $b \neq b^{\prime}$. Moreover $A_{j} b \subset U_{j}(B, C)$; so if $j \neq j^{\prime}$ then $A_{j} b \cap A_{j^{\prime}} b^{\prime}=\varnothing$ for any $b, b^{\prime} \in B$. Thus $A b \cap A b^{\prime}=\varnothing$ for $b \neq b^{\prime}$, and since $A$ was arbitrary, $S b \cap S b^{\prime}=\varnothing$ so $S$ is semisimple.

We conclude that to construct an arbitrary $r$-semisimple band from $r$-semisimple bands whose semilattice is simply ordered, we need only be concerned with preserving associativity as in Theorem 24 .

It is clear that an $r$-semisimple band is determined by the underlying semilattice, the right zero semigroups, the congruences $\tau_{A B}$ and the multiplication mappings. To make this somewhat more explicit we conclude with the following theorem. 
THEOREM 26. Let $S$ and $S^{\prime}$ be r-semisimple bands with $\theta: S \rightarrow S^{\prime}$ one-to-one and onto. We use primes to denote the image of both elements and subsets of $S$. Then $\theta$ is an isomorphism if and only if the following hold:

(1) The induced mapping on the semilattices and the restrictions to the right zero semigroups are isomorphisms.

(2) For any pair $A>C$ of right zero semigroups in $S$ and any index $k$, we have $U_{k}(A, C)^{\prime}=U_{k^{\prime}}\left(A^{\prime}, C^{\prime}\right)$ and $\phi_{k}(A, C) \theta=\theta \phi_{k^{\prime}}\left(A^{\prime}, C^{\prime}\right)$.

Proof. Suppose $\theta$ satisfies the given conditions. On any simply ordered chain of right zero semigroups the fact that $\theta$ is a homomorphism follows directly from (2). Suppose $A, B, C$ are right zero semigroups of $S$ with $A B, B A \subset C$ as in Theorem 24, and let $a \in A, b \in B$. By Theorem 24 there exists a $U_{j}(B, C)$ such that $C a \subset U_{j}(B, C)$ and a $U_{j *}\left(B^{\prime}, C^{\prime}\right)$ such that $C^{\prime} a^{\prime} \subset U_{j *}\left(B^{\prime}, C^{\prime}\right)$. Then $C^{\prime} a^{\prime}=(C a)^{\prime} \subset U_{j}(B, C)^{\prime}$ so that $U_{j^{\prime}}\left(B^{\prime}, C^{\prime}\right)=U_{j}(B, C)^{\prime}=U_{j *}\left(B^{\prime}, C^{\prime}\right)$. Thus $(a b)^{\prime}=\left(b \phi_{j}(B, C)\right)^{\prime}=b^{\prime} \phi_{j^{\prime}}\left(B^{\prime}, C^{\prime}\right)$ $=a^{\prime} b^{\prime}$ and $\theta$ is a homomorphism on $S$. We omit the proof of the converse.

\section{Semilattices of right zero semigroups.}

THEOREM 27. Let $S$ be a simply ordered semilattice of right zero semigroups. Then $S$ is $a$ band if and only if the following hold:

(1) For each pair $A>B$ of right zero semigroups in $S$ we have an equivalence relation $\tau_{A B}$ on $B$ with equivalence classes $U_{i}(A, B), i$ in some index set.

(2) For each $i$ we have a mapping $\phi_{i}(A, B): A \rightarrow U_{i}(A, B)$ with multiplication given by $a b=b$ and $b a=a \phi_{i}(A, B)$ for $a \in A, b \in U_{i}(A, B)$.

(3) If $A>B>C$, then $\tau_{A C} \leqq \tau_{B C}$, and for each pair $U_{j}(B, C), U_{i}(A, B)$ there exists a $U_{k}(A, C) \subset U_{j}(B, C)$ such that $U_{j}(B, C) U_{i}(A, B) \subset U_{k}(A, C)$. Moreover for this triple of indices $\phi_{i}(A, B) \phi_{j}(B, C)=\phi_{k}(A, C)$.

Proof. The proof is essentially the same as that of Theorem 21. The main thing to observe is that in (2) the mappings are not necessarily one-to-one if $S$ is a band, and furthermore that condition is sufficient to determine the multiplication for an arbitrary band of this type.

This theorem for a simply ordered semilattice together with Theorem 24 gives a characterization for an arbitrary semilattice of right zero semigroups. Finally we remark that the underlying semilattice, the right zero semigroups, the equivalence relations and the multiplication mappings determine these bands up to isomorphism. In this connection Theorem 26 carries over verbatim for any band which is a semilattice of rectangular bands.

\section{BIBLIOGRAPHY}

1. A. H. Clifford and G. B. Preston, Algebraic theory of semigroups, Vol. 1, Math Surveys No. 7, Amer. Math. Soc., Providence, R. I., 1961.

2. R. A. Dean and R. H. Oehmke, Idempotent semigroups with distributive right congruence lattices, Pacific J. Math. 14 (1964), 1187-1209. 
3. H. J. Hoehnke, Structure of semigroups, Canad. J. Math. 18 (1966), 449-491.

4. J. M. Howie, Naturally ordered bands, Glasgow Math. J. 8 (1967), 55-58.

5. N. Kimura, The structure of idempotent semigroups, Pacific J. Math. 8 (1958), 257-275.

6. D. McLean, Idempotent semigroups, Amer. Math. Monthly 61 (1954), 110-113.

7. R. H. Oehmke, On maximal congruences and finite semisimple semigroups, Trans. Amer. Math. Soc. 125 (1966), 223-237.

\section{UNIVERSITY OF MISSOURI,}

Columbia, Missouri 